\title{
Method to distinguish ferroelectric from nonferroelectric origin in case of resistive switching in ferroelectric capacitors
}

H. Kohlstedt, A. Petraru, K. Szot, A. Rüdiger, P. Meuffels, H. Haselier, R. Waser, and V. Nagarajan

Citation: Appl. Phys. Lett. 92, 062907 (2008);

View online: https://doi.org/10.1063/1.2841917

View Table of Contents: http://aip.scitation.org/toc/apl/92/6

Published by the American Institute of Physics

\section{Articles you may be interested in}

Resistive switching in metal-ferroelectric-metal junctions

Applied Physics Letters 83, 4595 (2003); 10.1063/1.1627944

Switchable diode effect and ferroelectric resistive switching in epitaxial $\mathrm{BiFeO}_{3}$ thin films Applied Physics Letters 98, 192901 (2011); 10.1063/1.3589814

Tunneling electroresistance in ferroelectric tunnel junctions with a composite barrier Applied Physics Letters 95, 052902 (2009); 10.1063/1.3195075

Tunneling electroresistance effect in a $\mathrm{Pt} / \mathrm{Hf}_{0.5} \mathrm{Zr}_{0.5} \mathrm{O}_{2} / \mathrm{Pt}$ structure

Applied Physics Letters 110, 093106 (2017); 10.1063/1.4977028

Resistive switching phenomena: A review of statistical physics approaches

Applied Physics Reviews 2, 031303 (2015); 10.1063/1.4929512

Ferroelectricity in hafnium oxide thin films

Applied Physics Letters 99, 102903 (2011); 10.1063/1.3634052

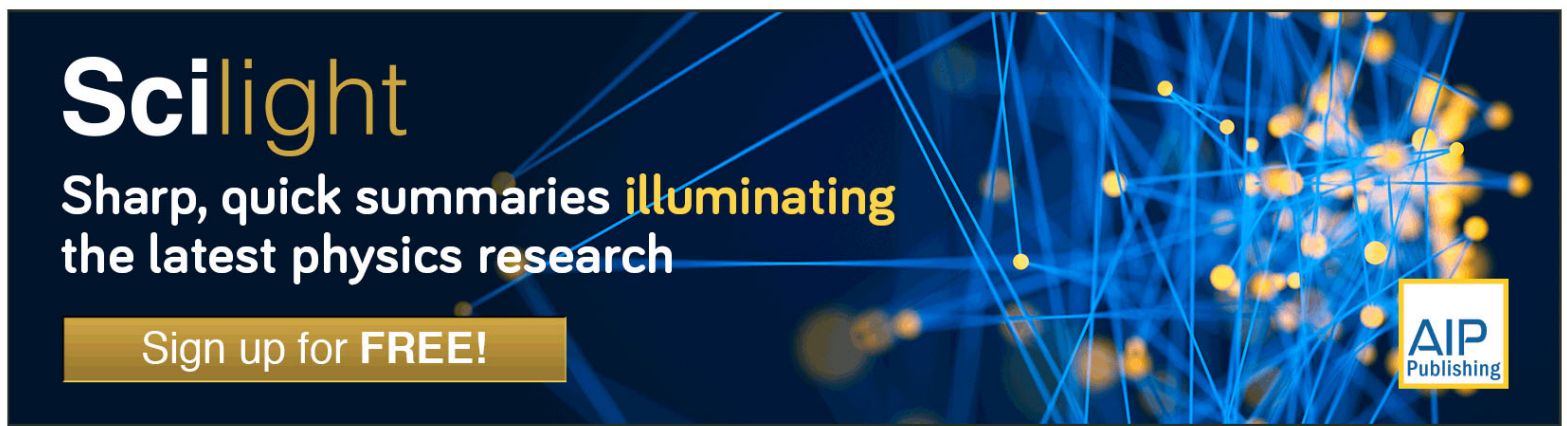




\title{
Method to distinguish ferroelectric from nonferroelectric origin in case of resistive switching in ferroelectric capacitors
}

\author{
H. Kohlstedt, ${ }^{1, a)}$ A. Petraru, ${ }^{1}$ K. Szot, ${ }^{1}$ A. Rüdiger, ${ }^{1}$ P. Meuffels, ${ }^{1}$ H. Haselier, ${ }^{1}$ R. Waser, ${ }^{1}$ \\ and V. Nagarajan ${ }^{2}$ \\ ${ }^{1}$ Institut für Festkörperforschung and the CNI, Forschungszentrum Jülich GmbH, Jülich 52425, Germany \\ ${ }^{2}$ School of Materials Science and Engineering, UNSW Sydney, NSW 2052, Australia
}

(Received 10 August 2007; accepted 16 January 2008; published online 14 February 2008)

\begin{abstract}
We present investigations on the resistive switching effect in $\mathrm{SrRuO}_{3} / \mathrm{PbZr}_{0.2} \mathrm{Ti}_{0.8} \mathrm{O}_{3} / \mathrm{Pt}$ ferroelectric capacitors. Using a conductive atomic force microscope, the out-of-plane piezoelectric response and the capacitive and resistive current were simultaneously measured as a function of applied bias voltage. We observed two independent switching phenomena, one attributed to the ferroelectric switching process and the other to resistive switching. We show that $I-V$ curves alone are not sufficient in ferroelectric materials to clarify the underlying switching mechanism and must be used with sufficient caution. (C) 2008 American Institute of Physics. [DOI: 10.1063/1.2841917]
\end{abstract}

Resistive switching is a well-known phenomenon in metal-insulator-metal (MIM) junctions observed in various material classes including amorphous, ${ }^{1,2}$ micro-crystalline, ${ }^{3}$ and crystalline insulators, ${ }^{4}$ solid state electrolytes, ${ }^{5}$ polymers, ${ }^{6,7}$ and as well as chalcogenides. ${ }^{8,9}$ Recently, it has also been observed in complex oxide compounds. ${ }^{10-13}$ It is noteworthy that some of these complex oxide compounds contain components such as $\mathrm{Ti}_{x} \mathrm{O}_{y}$ or $\mathrm{ZrO}_{2}$, which already exhibit switching alone. Recently, the role of oxygen ions for resistive switching of bulk $\mathrm{SrTiO}_{3}$ single crystals and the filamentary nature of the switching process were investigated. $^{14}$

Materials that display this behavior are now being explored for memory applications because the on/off resistance ratio can be very large, which makes them attractive as nonvolatile storage elements. Depending on the specific MIM and experimental parameters, several switching mechanisms need to be distinguished. ${ }^{15-19}$ It should be pointed out that despite the considerable progress in recent years, some of the mechanisms are not yet understood in detail. For the context of this paper, it is important to note that the topmost classification of the switching mechanisms concerns two different schemes with respect to the electrical polarity required. Switching is called unipolar when the switching procedure does not depend on the polarity of the voltage and current signal, and it is called bipolar when switching to a lowresistance state occurs at one voltage polarity and switching back to a high-resistance state takes place at the opposite polarity. In this letter, we only deal with the bipolar switching class. Resistive switching has been observed in ferroelectric materials by several groups. ${ }^{20-28}$ In the case of ferroelectrics, the issue is further complicated by characteristics such as interface properties, i.e., the barrier height and the sign of the remnant charge of the ferroelectric material. ${ }^{20-23,27,28}$ Nonetheless, from $I-V$ characteristics alone it might be a risky task to conclude the nature of the underlying mechanism. ${ }^{19,26,28,30}$ Therefore, it is critical to be able to distinguish a ferroelectric switching from that of a nonferroelectric origin, and this forms the motivation for this letter.

\footnotetext{
${ }^{\text {a) }}$ Author to whom correspondence should be addressed. Electronic mail: h.h.kohlstedt@fz-juelich.de
}

Eventually, one can think of two different strategies to verify a possible ferroelectric nature for resistive switching. The first and rather obvious approach is to measure the $I-V$ curve across the ferroelectric phase transition. In the paraelectric state, we expect no switching, whereas, by lowering the temperature below $T_{c}$, the resistive switching should appear. Here, $T_{c}$ is the critical temperature of the ferroelectric. Although this approach is the most convincing, there are several practical concerns. First, it would be necessary to have a second independent experimental method (e.g., a structural method) to determine $T_{c}$. Second and more severe, it is known that the phase transition in thin ferroelectric film can be rather broad and would make the interpretation of the findings questionable.

Therefore, we propose a different approach, i.e., to measure a ferroelectric parameter, in our case the piezoelectric constant $d_{33}$, while simultaneously monitoring the current transport (resistive component) through the device. A similar way was applied by Bune et al. by comparing resistive switching and pyroelectric currents in ferroelectric polymer capacitors. ${ }^{22}$ By comparing the ferroelectric coercive field $E_{c}$ with that needed to switch the device resistance $E_{s}$, we can easily deduce whether ferroelectricity and resistive switching are correlated. The case where $E_{c}=E_{s}$ would be a strong indicator that both effects are related, or a rather fortuitous case that the same field is needed, although both mechanisms are not correlated. On the other hand, if $E_{c} \neq E_{s}$ it means that ferroelectricity and resistive switching in the investigated devices are independent effects.

Epitaxial heterostructures $\mathrm{SrTiO}_{3}$ (substrate)/ $\mathrm{SrRuO}_{3}$ (base electrode) $/ \mathrm{Pb}\left(\mathrm{Zr}_{0.2} \mathrm{Ti}_{0.8}\right) \mathrm{O}_{3}$ using a high oxygen pressure sputtering setup were used for the investigations. More details about film growth and device fabrication can be found in Ref. 31. For the measurements, we used a conductive atomic force microscope in contact mode and the outof-plane deformation of the film due to applied electrical field was detected, which is related to the piezoelectric coefficient $d_{33}$ of the ferroelectric film via the inverse piezoelectric effect. The excitation signal supplied by a wave function generator was sinusoidal with a frequency of $1 \mathrm{kHz}$, an amplitude of $700 \mathrm{mV}_{\mathrm{PP}}$, and a variable bias in the range $\pm 2 \mathrm{~V}$. Thus, piezoelectric response loops were measured with a first 


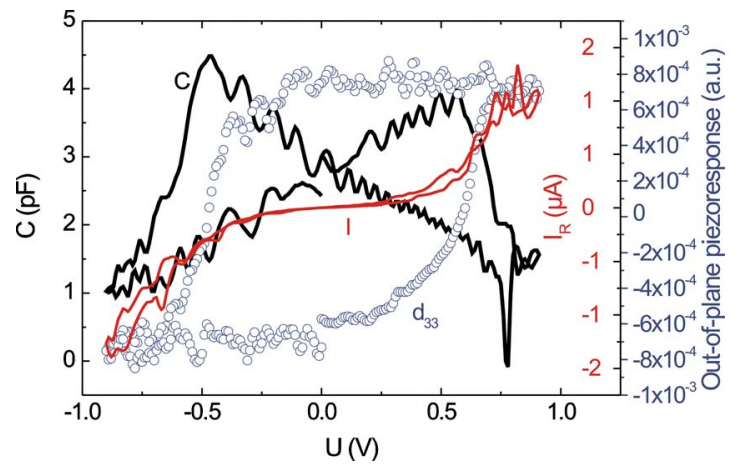

FIG. 1. (Color online) Simultaneous measurements of the resistive current through the ferroelectric sample, capacitance, and its piezoelectric response for a pad of $7 \mu \mathrm{m}^{2}$ on a $30 \mathrm{~nm}$ thick PZT film. The piezoelectric $d_{33}$ loop and the $C-V$ characteristic appear very clearly, indicating that the film is ferroelectric.

lock-in amplifier. Simultaneously, the dynamic resistance of the sample was measured by a second lock-in amplifier. More details on the experimental setup can be found elsewhere. ${ }^{32}$ Figure 1 shows simultaneous measurements of the $d_{33}$ loop, the resistive current through the sample $I_{R}$, and the capacitance $C$ (double-humped loop) as a function of applied bias $V$ of an as-grown sample. The change in sign of the piezoelectric coefficient $d_{33}$ is specific for ferroelectric materials and it takes place when the coercive field is reached (here approximately $\pm 167 \mathrm{kV} / \mathrm{cm}$ ). The bias was varied in the range $-0.9-+0.9 \mathrm{~V}$. No noticeable change at this field is observed in the resistive current. In addition, the $C-V$ butterfly is shown in Fig. 1. The coercive field from the $C$ - $V$ loops coincides with that of the $\mathrm{d}_{33}$ vs $V$ loops. Therefore, the ferroelectric character of the capacitors is evident. By further increasing the voltage amplitude, we observed resistive switching at approximately +1 and $-1 \mathrm{~V}$ for this particular sample (at $\pm 330 \mathrm{kV} / \mathrm{cm}$ ). The results are presented in Fig. 2. Surprisingly, the deflection signal also followed fairly well the current transport curve. This effect can be misinterpreted as a giant increase in the piezoelectric coefficient. A closer look tells us that at $0.7 \mathrm{~V}$ a current of $22 \mu$ A flows through the atomic force microscopy (AFM) tip contact area of approximately $3 \times 10^{-12} \mathrm{~cm}^{2}$. This yields a current density of about $7 \times 10^{7} \mathrm{~A} / \mathrm{cm}^{2}$, and at such high current densities, local joule heating is not negligible and

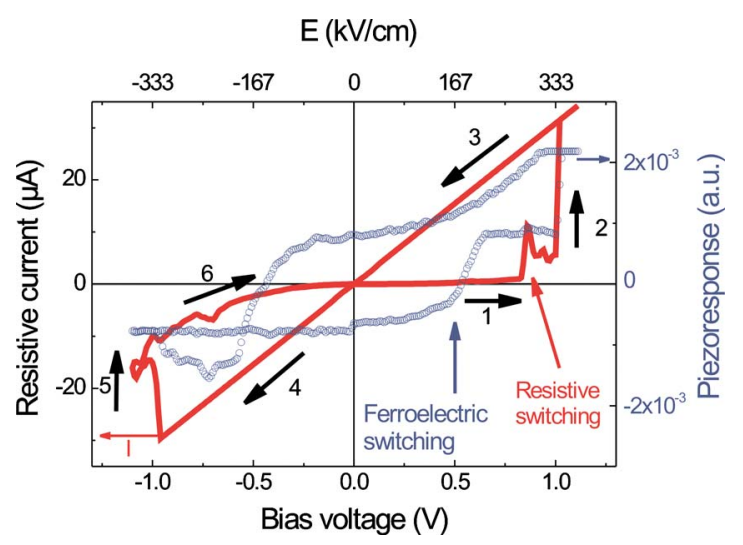

FIG. 2. (Color online) Simultaneous measurements of the resistive current through the ferroelectric sample and the piezoelectric response. The ferroelectric switching can be distinguished from the $d_{33}$ loop and appears at an applied electic field of about $167 \mathrm{kV} / \mathrm{cm}$, while the resistive switching can be observed at about $330 \mathrm{kV} / \mathrm{cm}$ applied electric field. (a)

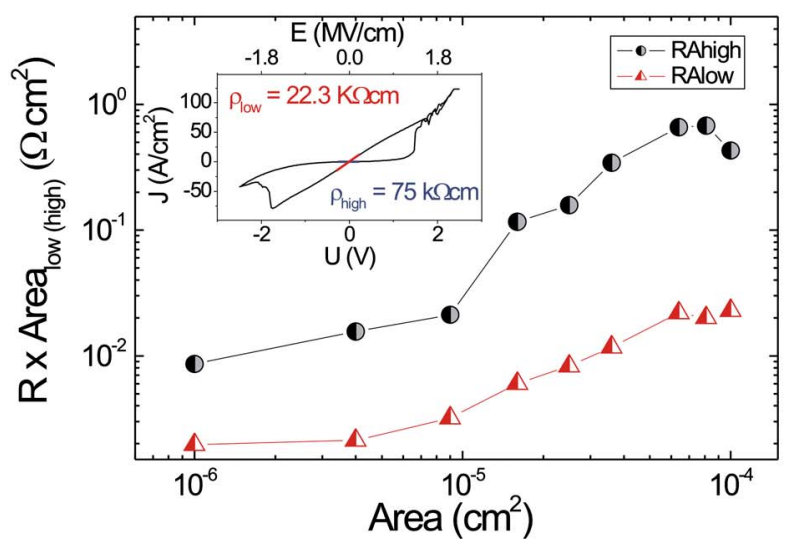

(b)

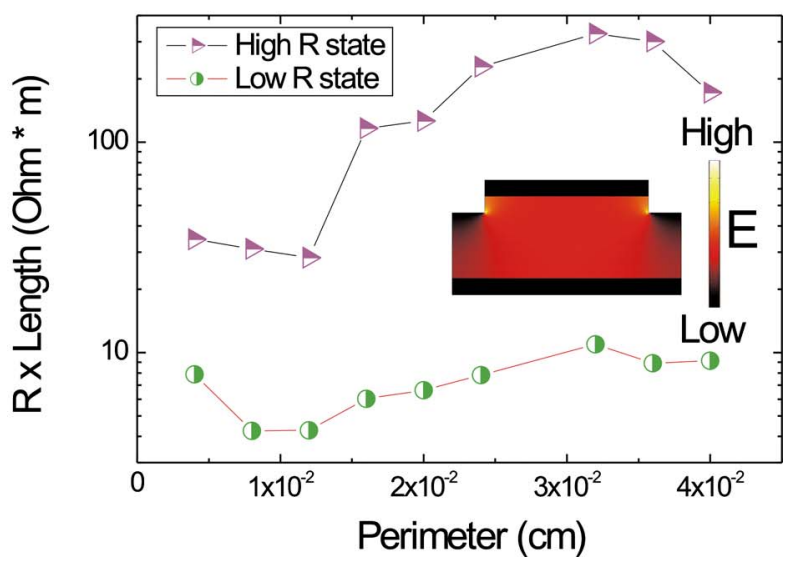

FIG. 3. (Color online) (a) Resistance area $(R \times A)$ product for a $9 \mathrm{~nm}$ thick $\mathrm{PbZr}_{20} \mathrm{Ti}_{80} \mathrm{O}_{3}$ film for the low and high ohmic states. The resistance was determined with a linear fit around origin from the $I-V$ characteristics $( \pm 100 \mathrm{mV})$ around the origin. The inset graph shows an $I-V$ characteristic including the resistive switching effect of a $90 \times 90 \mu \mathrm{m}^{2}$ device. (b) Resistance perimeter $(R \times l)$ of a capacitor. The inset shows the electric field distribution.

could create abnormal behavior. We have verified this by replacing the ferroelectric sample with a Si diode connected between two Pt electrodes on a substrate. We gradually increased the forward bias and when the current has exceeded $10 \mu \mathrm{A}$, Joule heating effects started to influence the piezoresponse measurement. Thus, deflections that are recorded when currents larger than $10 \mu \mathrm{A}$ flow through the AFM tip are considered in the context of this letter as a measurement artifact.

At voltages below $0.8 \mathrm{~V}$, the current through the tip is so low that no significant heat was generated. Thus, without doubt in this regime, the real $d_{33}$ vs $V$ was observed, showing the $d_{33}$ hysteresis loops specific for ferroelectric thin films. These results show that in these samples, ferroelectricity is a phenomenon independent from the resistive switching effect because $E_{s} \cong 2 E_{c}$. To further investigate the resistive switching, we fabricated capacitors with large variation in size on a similar ferroelectric film having a nominal thickness of $9 \mathrm{~nm}$. $I-V$ measurements at a frequency of $10 \mathrm{~Hz}$ (linear ramp driving signal) were performed for a large range of electrode areas from 1 to $10^{4} \mu \mathrm{m}^{2}$. A commercially available instrument (AIXACCT thin film analyzer) was used. A typical $I-V$ curve is shown by the inset in Fig. 3(a). In the regions around the origin $( \pm 100 \mathrm{mV})$, we used linear fitting to extract the values of the low and high resistance states of each 
capacitor. The area dependency $R \times A$ vs $A$ is shown in Fig. $3(\mathrm{a})$. The finite slope of this curve clearly indicates an inhomogeneous current flow through the device. With a larger area, the $R \times A$ product increases, which means that the current conducted is independent of the geometric area of the MIM device. In other words, it is a local conduction mechanism rather than a bulk one. In case of a homogenous bulk or interface effect, one expects $R \times A$ not to be a function of $A$.

To further investigate the location of such filaments underneath the top electrode, we plotted the $R \times l$ product versus $l$ [see Fig. 3(b)]. Here, $l$ is the perimeter of a capacitor. This approach was motivated by the following facts: The perimeter of our structures is subject to two contributions that possibly favor switching along the edges. First, the etching process weakens the underlying perovskite along the top electrode, leaving it modified and, therefore, more susceptible to electroformation. Second, the geometry of the top electrode causes a significantly enhanced field along its perimeter, an effect well known from dielectric degradation and ferroelectric domain nucleation. ${ }^{33}$ The inset of Fig. 3(b) illustrates the vertical projection of the electric field between top and bottom electrode in a dielectric. In contrast to the first effect, the latter is omnipresent with considerable variations depending on the exact contact geometry.

Further, the resistive switching effect is much more pronounced for large area pads (exceeding $7 \mu \mathrm{m}$ in lateral size), and it appears at a relatively low applied electric field. In contrast, for small area pads with sizes below $3 \mu \mathrm{m}$, the resistive switching effect is less pronounced; i.e., the ratio between the high and low resistance decreases and appears at higher values of the applied voltage. For pads smaller than $1 \mu \mathrm{m}$ in size, the resistive switching is rather difficult to observe and the increase of the applied electric field far over the dielectric breakdown strength still does not yield a favorable resistance switching effect. When the size of the device is in the range of 1 micron and lower, the probability of finding a defect decreases drastically. This, in turn, would affect the switching mechanism and, as a consequence, lower the $R_{\text {on/roff }}$ ratio significantly. Nonetheless, the experiments showed that local conductive channels are likely to be formed while applying high enough voltage to the capacitors. We conclude that resistive switching observed in Ref. 26 is most likely not caused by ferroelectricity. However, we would like to point out that our results do not necessarily mean that a ferroresistive switch could not exist at all. ${ }^{29,34,35}$ We demonstrate that $I-V$ curves alone are not sufficient to understand the switching mechanism dominating the $I-V$ characteristics.

We thank the Volkswagen-Stiftung under Contract No. I/77737 and the Deutsche Forschungsgemeinschaft for financial support. V. N. acknowledges funding from the ARC Discovery Project DP0666231.

${ }^{1}$ J. W. Park, D. Y. Kim, and J. K. Lee, J. Vac. Sci. Technol. A 23, 1309 (2005).

${ }^{2}$ H. Kohlstedt, K. H. Gundlach, and S. Kuriki, J. Appl. Phys. 73, 2564 (1993).

${ }^{3}$ B. J. Choi, D. S. Jeong, S. K. C. Rohde, S. Choi, J. H. Oh, H. J. Kim, and C. S. Hwang, J. Appl. Phys. 98, 033715 (2005).

${ }^{4}$ Y. Watanabe, J. G. Bednorz, A. Bietsch, Ch. Gerber, D. Widmer, A. Beck, and S. J. Wind, Appl. Phys. Lett. 78, 3738 (2001).

${ }^{5}$ M. N. Kozicki, M. Park, and M. Mitkova, IEEE Trans. Nanotechnol. 4, 331 (2005)

${ }^{6}$ L. Ma, S. Pyo, J. Ouyang, Q. Xu, and Y. Yang, Appl. Phys. Lett. 82, 1419 (2003).

${ }^{7}$ M. A. Reed, J. Chen, A. M. Rawlett, D. W. Price, and J. M. Tour, Appl. Phys. Lett. 78, 3735 (2003).

${ }^{8}$ A. L. Greer and N. Mathur, Nature (London) 437, 1246 (2005).

${ }^{9}$ S. R. Ovshinsky, Phys. Rev. Lett. 21, 1450 (1968).

${ }^{10}$ C. Rossel, G. I. Meijer, D. Brémaud, and D. Widmer, J. Appl. Phys. 90, $2892(2001)$.

${ }^{11}$ A. Beck, J. G. Bednorz, Ch. Gerber, C. Rossel, and D. Widmer, Appl. Phys. Lett. 77, 139 (2000).

${ }^{12}$ R. Oligschlaeger, R. Waser, R. Meyer, S. Karthäuser, and R. Dittmann, Appl. Phys. Lett. 88, 042901 (2006).

${ }^{13}$ A. Sawa, T. Fuji, M. Kawasaki, and T. Tokura, Appl. Phys. Lett. 85, 4073 (2004).

${ }^{14}$ K. Szot, G. Bihlmeyer, W. Speier, and R. Waser, Nat. Mater. 5, 312 (2006).

${ }^{15}$ G. Dearnaley, Phys. Lett. A 25, 760 (1967).

${ }^{16}$ T. W. Hickmott and W. R. Hiatt, Solid-State Electron. 13, 1033 (1970).

${ }^{17}$ J. G. Simmons and R. Verderber, Appl. Phys. Lett. 10, 197 (1967).

${ }^{18}$ G. Dearnaley, A. M. Stoneham, and D. V. Morgan, Rep. Prog. Phys. 33, 1129 (1970).

${ }^{19}$ R. Waser and M. Aono, Nat. Mater. 6, 833 (2007).

${ }^{20}$ P. W. M. Blum, R. M. Wolf, J. F. M. Gilessen, and M. P. C. M. Krijin, Phys. Rev. Lett. 73, 2107 (1994).

${ }^{21}$ Y. Watanabe, Appl. Phys. Lett. 66, 28 (1995).

${ }^{22}$ A. Bune, S. Ducharme, V. Fridkin, L. Blinov, S. Palto, N. Petukhova, and S. Yudin, Appl. Phys. Lett. 67, 3975 (1995).

${ }^{23}$ K. Gotoh, H. Tamura, H. Takauchi, and A. Yoshida, Jpn. J. Appl. Phys., Part 2 35, 39 (1996).

${ }^{24}$ M. Okano and Y. Watanabe, Appl. Phys. Lett. 76, 233 (2000).

${ }^{25}$ Y. Watanabe, Phys. Rev. B 59, 11257 (1999).

${ }^{26}$ J. Rodríguez Contreras, H. Kohlstedt, U. Poppe, R. Waser, and Ch. Buchal, Appl. Phys. Lett. 83, 126 (2003).

${ }^{27}$ S. Y. Wu, H. X. Liu, L. Gu, R. K. Singh, L. Budd, M. van Schilfgaarde, M. R. McCartney, D. J. Smith, and N. Newman, Appl. Phys. Lett. 82, 3047 (2003).

${ }^{28}$ R. Meyer and H. Kohlstedt, Integr. Ferroelectr. 64, 77 (2004).

${ }^{29}$ R. Meyer and H. Kohlstedt, IEEE Trans. Ultrason. Ferroelectr. Freq. Control 53, 2340 (2006).

${ }^{30}$ P. van der Sluis, Appl. Phys. Lett. 82, 4089 (2003); 84, 2211 (2004).

${ }^{31}$ J. Rodriguez Contreras, H. Kohlstedt, A. Petraru, A. Gerber, B. Hermanns, H. Haselier, H. V. Nagarajan, and C. Buchal, J. Cryst. Growth 277, 210 (2005).

${ }^{32}$ A. Petraru, V. Nagarajan, H. Kohlstedt, R. Ramesh, D. G. Schlom, and R. Waser, Appl. Phys. A: Mater. Sci. Process. 84, 67 (2006).

${ }^{33}$ M. Dawber, D. J. Jung, and J. F. Scott, Appl. Phys. Lett. 82, 436 (2003).

${ }^{34}$ R. Meyer and R. Waser, J. Appl. Phys. 100, 051611 (2006).

${ }^{35}$ E. Y. Tsymbal and H. Kohlstedt, Science 313, 181 (2006). 